\title{
Induced gravity on intersecting brane worlds: Maximally symmetric solutions
}

\author{
Olindo Corradini, ${ }^{1, *}$ Kazuya Koyama, ${ }^{2, \dagger}$ and Gianmassimo Tasinato ${ }^{3, \$}$ \\ ${ }^{1}$ Dipartimento di Fisica, Università di Bologna and INFN Sezione di Bologna, Via Irnerio, 46-Bologna I-40126, Italy \\ ${ }^{2}$ Institute of Cosmology and Gravitation, University of Portsmouth, Portsmouth PO1 2EG, United Kingdom \\ ${ }^{3}$ Instituto de Fisica Teorica, UAM/CSIC, Facultad de Ciencias C-XVI, C.U. Cantoblanco, E-28049-Madrid, Spain
}

(Received 11 December 2007; published 7 April 2008)

\begin{abstract}
We explore models of intersecting brane worlds with induced gravity terms on codimension-one branes and on their intersection. Maximally symmetric solutions for the branes and the intersection are found. We find new self-accelerating solutions. In a 6D spacetime, the solutions realize the seesaw modification of gravity where the UV scale of the modification to $4 \mathrm{D}$ gravity is determined by $6 \mathrm{D}$ Planck scale given by $M_{6} \sim 10^{-3} \mathrm{eV}$ and the IR scale of the modification is determined by $M_{6}^{2} / M_{4} \sim H_{0} \sim 10^{-42} \mathrm{GeV}$, where $H_{0}$ is the present-day Hubble scale. We find that it is increasingly difficult to construct phenomenologically viable models in higher-dimensional spacetime due to the necessity to have the lower value for the fundamental Planck scale to realize the late time acceleration. It is found that the system also admits selftuning solutions where the tension at the intersection does not change the geometry of the intersection. The induced gravity terms can avoid the necessity to compactify the extra dimensions. Finally, we discuss the possibility to have ordinary matter at the intersection, without introducing any regularization, using the induced gravity terms.
\end{abstract}

DOI: 10.1103/PhysRevD.77.084006

PACS numbers: $04.50 .-\mathrm{h}$

\section{INTRODUCTION}

Brane-world models with large distance modification of Einstein gravity are invoked in scenarios that aim to geometrically describe present-day acceleration, without introducing dark energy [1-3] (for a review see [4]). A celebrated example is the Dvali-Gabadadze-Porrati (DGP) model in a 5D spacetime [1]. The brane action includes a quantum-induced Einstein-Hilbert $(\mathrm{EH})$ term that recovers 4D gravity on small scales. This model realizes a so-called self-accelerating solution that features a 4D de Sitter phase even though the 3-brane is completely empty. However, so far, only codimension-one examples of such solutions have been proposed and these backgrounds are known to suffer from ghost instabilities [5]. An interesting possibility would thus be to look for other such solutions in higher codimension setups, that might lead to ghost-free models.

On the other hand, it is known that the presence of more than one extra dimension might offer better possibilities for the solution of the (particle physics) cosmological constant problem. In fact, codimension-two brane worlds with finite [6-9] and infinite [10,11] volume extra space have been extensively studied in the past few years towards a solution of the cosmological constant problem. ${ }^{1} \mathrm{~A}$ common feature of all these models is that the energymomentum tensor on a codimension-two brane only controls the global properties of the background geometry

\footnotetext{
*corradini@bo.infn.it

†Kazuya.Koyama@port.ac.uk

‡ianmassimo.tasinato@uam.es

${ }^{1}$ See [12] for attempts in this direction in the codimension-one case.
}

without affecting the local structure of the brane itself which remains flat. A realization of this idea is called the self-tuning solution of the cosmological constant problem. Unfortunately, in the finite volume case, consistency conditions associated to the quantization condition of a background flux which is introduced to compactify the extra-dimensional space are shown to be problematic and the self-tuning mechanism in its simplest form does not work [13]. More in general, another delicate feature of codimension-two brane models (both with finite- and infinite-volume) is related to the fact that only the energy-momentum tensor of the form of pure tension can be accommodated on a thin radially symmetric codimension-two brane, to ensure the regularity of the background metric at the brane position [14]. This last problem can be ameliorated by including higher order Gauss-Bonnet terms for gravity in the bulk [15]; however, other difficulties arise when one tries to embed an isotropic and homogeneous fluid on such a radially symmetric purely conical codimension-two brane [16]. This suggests the necessity of regularizing the brane and allowing it to acquire some structure. For the finite volume scenario, this approach was started by [17] that shows how normal gravity can be recovered in this context, and it is further developed by various other works [18]. ${ }^{2}$ The regularization of the codimension-two brane with induced gravity has been also studied intensively [20,21].

In this paper, we take a different route to tackle this regularization problem. We consider a system in which the brane is a pure codimension-two object, without an internal

\footnotetext{
${ }^{2}$ See also [19] for articles discussing gravity in higher codimensions.
} 
structure, but with induced gravity terms on it. This system, as we already mentioned at the beginning, is by itself interesting to investigate, for finding new self-accelerating configurations. The induced gravity terms can avoid the necessity to compactify the higher-dimensional space, by providing a mechanism to obtain four-dimensional gravity in the relevant regimes with infinite-volume extra dimensions. Consequently, the bulk gauge field is no more needed, and this suggests to reconsider the self-tuning mechanism in this context.

The model that we consider is a codimension-two brane, that lies at the intersection of two codimension-one branes embedded in a six-dimensional space. This system was studied in the context of standard gravity [22] and GaussBonnet gravity [23] elaborating an idea developed in [24]. ${ }^{3}$ A model with generic angle between two intersecting branes, the so-called Origami world, was first considered in [26]. In our case, we consider a situation in which induced gravity terms are allowed on the codimension-one branes and also at the intersection. We study various maximally symmetric configurations of branes and explore configurations that exhibit self-tuning or self-accelerating properties. Let us also point out that the presence of induced Einstein-Hilbert terms on intersecting codimensionone branes might allow for generic localized matter on the intersection and thus allow for generic FriedmanRobertson-Walker (FRW) cosmology. We discuss this possibility in conclusions, briefly summarizing the results of a companion paper [27].

While we were preparing this work, a relevant paper [28] appeared that studied a nested 4D brane in a 5D codimension brane in a 6D spacetime by generalizing [29]. Although there are some similarities with our system, their model is different from ours as their 4D brane behaves like a vortex when gravity behaves as $6 \mathrm{D}$ while the intersection does not.

\section{MAXIMALLY SYMMETRIC CONFIGURATIONS OF BRANES}

We consider a system of two intersecting codimensionone branes embedded in a six-dimensional spacetime. They intersect on a four-dimensional codimension-two brane where observers like us can be localized. We take an Einstein-Hilbert action for gravity in the bulk and we allow for induced gravity terms on the codimension-one branes, as well as on the intersection. Besides gravity, we allow for a cosmological constant term in the bulk, $\Lambda_{B}$, and for additional fields localized on the branes described by general Lagrangians $L$ 's. The general action assumes the form

\footnotetext{
${ }^{3}$ Intersecting brane models are very important in string theory, for the possibility to build standardlike models with chiral matter [25].
}

$$
\begin{aligned}
S= & \int_{\text {bulk }} d^{6} x \sqrt{-g}\left(\frac{M_{6}^{4}}{2} R-\Lambda_{B}\right) \\
& +\sum_{i=1}^{2} \int_{\Sigma_{i}} d^{5} x \sqrt{-g_{(i)}}\left(\frac{M_{5, i}^{3}}{2} R_{(i)}+L_{(i)}\right) \\
& +\int_{\Sigma_{n}} d^{4} x \sqrt{-g_{ก}}\left(\frac{M_{4}^{2}}{2} R_{\cap}+L_{\cap}\right),
\end{aligned}
$$

where $\Sigma_{\cap} \equiv \bigcap_{i} \Sigma_{i}$ denotes a three-brane at the intersection between all codimension-one four-branes $\Sigma_{i}$. We can have different fundamental scales in the different regions of the space, $M_{6}, M_{5, i}$, and $M_{4}$. The induced gravity terms could be generated, as it was proposed in the original model, by quantum corrections from matter loops on the brane. It is also interesting to note that induced curvature terms appear quite generically in junction conditions of higher codimension branes when considering natural generalizations of Einstein gravity $[15,19]$ as well as in string theory compactifications [30], orientifold models, and intersecting Dbrane models [31].

In this paper, we focus on a configuration with static codimension-one branes. They are characterized by tensions $\Lambda_{1}$ and $\Lambda_{2}$, while the intersection has tension $\lambda$. The branes intersect with a generic angle, along the lines of Origami-world [26]. The six-dimensional bulk is characterized by a maximally symmetric geometry

$$
\begin{aligned}
d s^{2} & =A^{2}\left(t, z^{1}, z^{2}\right)\left(\eta_{\mu \nu} d x^{\mu} d x^{\nu}+\delta_{k h} d z^{k} d z^{h}\right), \\
A\left(t, z^{1}, z^{2}\right) & =\frac{1}{H t+k_{i} z^{i}} .
\end{aligned}
$$

Also the codimension-one branes and their intersection are characterized by maximally symmetric geometries. We are going to relate the Hubble parameters on the branes with the geometrical parameters that control our system.

The parameters $H$ and $k_{i}$ appearing in the warp factor $A$ satisfy the following relation:

$$
\frac{\Lambda_{B}}{10}=H^{2}-k_{1}^{2}-k_{2}^{2},
$$

in order to solve the Einstein equations in the bulk. The branes form a generic angle and are characterized by normal vectors

$$
\begin{gathered}
\mathbf{n}^{(1)}=\left(\sin \alpha_{1},-\cos \alpha_{1}\right), \\
\mathbf{n}^{(2)}=\left(\sin \alpha_{2}, \cos \alpha_{2}\right),
\end{gathered}
$$

in the two transverse space directions $z^{i}$, so that the angle between the two branes is $\alpha \equiv\left(\alpha_{1}+\alpha_{2}\right)$. We refer to the brane $\Sigma_{i}$ as orthogonal to the vector $\mathbf{n}^{(i)}$. It is useful to define new coordinates

$$
\tilde{z}^{k}=\mathbf{n}^{(k)} \cdot \mathbf{z} \quad \text { with } \mathbf{z}=\left(z^{1}, z^{2}\right),
$$

that allow us to define the brane positions as $\tilde{z}^{i}=0$. Following [26], we define new vectors $l_{(k)}$ as 


$$
\mathbf{l}_{(k)} \cdot \mathbf{n}^{(h)}=\delta_{k}^{h} \rightarrow\left\{\begin{array}{l}
\mathbf{l}_{(1)}=\frac{1}{\sin \alpha}\left(\cos \alpha_{2},-\sin \alpha_{2}\right), \\
\mathbf{l}_{(2)}=\frac{1}{\sin \alpha}\left(\cos \alpha_{1}, \sin \alpha_{1}\right),
\end{array}\right.
$$

that allow us to write

$$
\mathbf{z}=\tilde{z}^{k} \mathbf{l}_{(k)},
$$

so that

$$
\frac{\partial z^{h}}{\partial \tilde{z}^{k}}=l_{(k)}^{h}
$$

is the projection operator to the brane $\Sigma_{k}$. The two branes $\Sigma_{1}$ and $\Sigma_{2}$ are fixed points of $Z_{2}$ symmetries acting on the six-dimensional space. We wish to investigate the conditions that their tensions must satisfy in order to solve the Einstein equations. We will use the junction conditions obtained from the Israel formalism. We focus on the brane $\Sigma_{1}$ and find the extrinsic curvature at its position. Recall that the second brane $\Sigma_{2}$ is also a fixed point of $Z_{2}$ symmetry. To implement this information, we require the metric to be symmetric under $\tilde{z}^{2} \rightarrow-\tilde{z}^{2}$. The transverse space line element in the new coordinate frame changes from

$$
\delta_{i j} d z^{i} d z^{j}=d \mathbf{z} \cdot d \mathbf{z}
$$

to

$$
\begin{array}{r}
\mathbf{l}_{(1)} \cdot \mathbf{l}_{(1)} d \tilde{z}^{1} d \tilde{z}^{1}+2 \mathbf{l}_{(1)} \cdot \mathbf{l}_{(2)} \operatorname{sgn}\left(\tilde{z}^{2}\right) d \tilde{z}^{1} d \tilde{z}^{2} \\
+\mathbf{l}_{(2)} \cdot \mathbf{l}_{(2)} d \tilde{z}^{2} d \tilde{z}^{2}=\tilde{\gamma}_{m n} d \tilde{z}^{m} d \tilde{z}^{n},
\end{array}
$$

with

$$
\tilde{\gamma}_{m n}=\frac{1}{\sin ^{2} \alpha}\left(\begin{array}{cc}
1 & \cos \alpha \operatorname{sgn}\left(\tilde{z}^{2}\right) \\
\cos \alpha \operatorname{sgn}\left(\tilde{z}^{2}\right) & 1
\end{array}\right) .
$$

The bulk line element reads ${ }^{4}$

$$
\begin{gathered}
d s^{2}=A^{2}\left(t, \tilde{z}^{1},\left|\tilde{z}^{2}\right|\right)\left(\eta_{\mu \nu} d x^{\mu} d x^{\nu}+\tilde{\gamma}_{m n} d \tilde{z}^{m} d \tilde{z}^{n}\right), \\
A\left(t, \tilde{z}^{1},\left|\tilde{z}^{2}\right|\right)=\frac{1}{H t+\mathcal{C}_{1} \tilde{z}^{1}+\mathcal{C}_{2}\left|\tilde{z}^{2}\right|}, \\
\mathcal{C}_{1}=\frac{k_{1} \cos \alpha_{2}-k_{2} \sin \alpha_{2}}{\sin \alpha}, \\
\mathcal{C}_{2}=\frac{k_{1} \cos \alpha_{1}+k_{2} \sin \alpha_{1}}{\sin \alpha} .
\end{gathered}
$$

The inverse metric is given by

$$
g^{M N}=A^{-2}\left(\begin{array}{cc}
\eta^{\mu \nu} & 0 \\
0 & \tilde{\gamma}^{m n}
\end{array}\right)
$$

where

\footnotetext{
${ }^{4}$ We emphasize that we are imposing a $Z_{2}$ symmetry at the point $\tilde{z}_{2}=0$, while at this level we are not imposing a similar reflection symmetry at $\tilde{z}_{1}=0$. This last symmetry will be applied later at the end of this section, when using Israel formalism to find the junction conditions.
}

$$
\tilde{\gamma}^{m n}=\left(\begin{array}{cc}
1 & -\cos \alpha \operatorname{sgn}\left(\tilde{z}^{2}\right) \\
-\cos \alpha \operatorname{sgn}\left(\tilde{z}^{2}\right) & 1
\end{array}\right) .
$$

In the new coordinate frame the normal vector to the brane $\Sigma_{1}$ is

$$
\tilde{n}_{k}^{(1)}=\frac{\partial z^{h}}{\partial \tilde{z}^{k}} n_{h}^{(1)}=\mathbf{l}_{(k)} \cdot \mathbf{n}^{(1)}=\delta_{k}^{1}=(1,0) .
$$

The normal vector that has a unit length with respect to the bulk metric is

$$
\tilde{n}_{M}^{(1)}=A\left(0_{\mu}, 1,0\right) .
$$

The projection operator (9) allows us to define the induced metric on the brane $\Sigma_{1}$, whose $\left(\tilde{z}_{2}, \tilde{z}_{2}\right)$ component reads ${ }^{5}$

$$
g_{\tilde{z}_{2} \tilde{z}_{2}}^{(5)}=l_{\left(\tilde{z}_{2}\right)}^{k} l_{\left(\tilde{z}_{2}\right)}^{h} A^{2} \delta_{k h}=A^{2}\left(\mathbf{l}_{(2)}\right)^{2}=\frac{A^{2}}{\sin ^{2} \alpha} .
$$

Hence, the line element on the brane $\Sigma_{1}$ is

$$
d s_{\Sigma_{1}}^{2}=A^{2}\left(t, 0,\left|\tilde{z}^{2}\right|\right)\left(\eta_{\mu \nu} d x^{\mu} d x^{\nu}+\frac{d \tilde{z}_{2} d \tilde{z}_{2}}{\sin ^{2} \alpha}\right) .
$$

Each induced brane metric corresponds to a maximally symmetric space with a constant Hubble parameter. For the brane $\Sigma_{1}$, the Hubble parameter is given by

$$
H_{1}^{2}=H^{2}-\sin ^{2} \alpha \mathcal{C}_{2}^{2} \text {, }
$$

from which

$$
\sin \alpha \mathcal{C}_{2}=-\epsilon_{1} \sqrt{H^{2}-H_{1}^{2}},
$$

where $\epsilon_{1}$ is equal to plus or minus one and distinguishes two different branches of solutions. An analogous relation connects $H_{2}$ to $\mathcal{C}_{1}$. It is now straightforward to compute the components of the extrinsic curvature. Then, we can apply the formalism of the Israel junction conditions to extract information about the energy-momentum tensor on the brane. Particularly interesting is its $\left(\tilde{z}_{2}, \tilde{z}_{2}\right)$ component that reads

$$
K_{\tilde{z}_{2} \tilde{z}_{2}}=\tilde{\nabla}_{\tilde{z}_{2}} \tilde{n}_{\tilde{z}_{2}}=-g_{\tilde{z}_{2} \tilde{z}_{2}}\left(\mathcal{C}_{1}-\cos \alpha \mathcal{C}_{2}\right)+2 A \delta\left(\tilde{z}_{2}\right) \frac{\cos \alpha}{\sin ^{2} \alpha} .
$$

Defining $w_{2} \equiv \tilde{z}_{2} / \sin \left(\alpha_{1}+\alpha_{2}\right)$ in order to remove the $\sin \left(\alpha_{1}+\alpha_{2}\right)$ factor from the metric (21), one obtains

$$
K_{w_{2}}{ }^{w_{2}}=-\left(\mathcal{C}_{1}-\cos \alpha \mathcal{C}_{2}\right)+\frac{2}{A} \delta\left(w_{2}\right) \frac{\cos \alpha}{\sin \alpha},
$$

where we take into account the Jacobian in the delta function $\delta\left(\tilde{z}_{2}\right)=\delta\left(w_{2}\right) / \sin \alpha$.

It is important to realize that only this component of the extrinsic curvature contains a singular term $\delta\left(w_{2}\right)$, so that

\footnotetext{
${ }^{5}$ The induced metric is invariant under bulk diffeomorphisms. Using the bulk frame $\left(\tilde{z}_{1}, \tilde{z}_{2}\right)$, one simply gets $g_{\tilde{z}_{2} \tilde{z}_{2}}^{(5)}=$ $\delta_{\tilde{z}_{2}}^{m} \delta_{\tilde{z}_{2}}^{n} A^{2} \gamma_{m n}=A^{2} \gamma_{\tilde{z}_{2} \tilde{z}_{2}}$ that obviously agrees with the result obtained in the original $\left(z_{1}, z_{2}\right)$ frame.
} 
$K_{a}{ }^{b}-\delta_{a}{ }^{b} K$ has a singular contribution only along the four-dimensional coordinates that characterize the intersection. It must be compensated by the energy-momentum tensor of matter localized at the intersection itself. The maximally symmetric metric at the intersection reads

$$
d s_{4}^{2 \text { int }}=A^{2}(t, 0,0) \eta_{\mu \nu} d x^{\mu} d x^{\nu}
$$

with Hubble parameter $H$.

The Israel junction conditions relate the components of the extrinsic curvature to the energy-momentum tensor on the branes. A straightforward calculation using the relation between the $\mathcal{C}_{i}$ functions and the induced Hubble parameters leads to the following conditions: ${ }^{6}$

$$
\begin{gathered}
\Lambda_{1}=6 M_{5,1}^{3} H_{1}^{2}-\frac{8 M_{6}^{4}}{\sin \alpha}\left(\epsilon_{2} \sqrt{H^{2}-H_{2}^{2}}\right. \\
\left.-\epsilon_{1} \cos \alpha \sqrt{H^{2}-H_{1}^{2}}\right) \\
\Lambda_{2}=6 M_{5,2}^{3} H_{2}^{2}-\frac{8 M_{6}^{4}}{\sin \alpha}\left(\epsilon_{1} \sqrt{H^{2}-H_{1}^{2}}\right. \\
\left.-\epsilon_{2} \cos \alpha \sqrt{H^{2}-H_{2}^{2}}\right), \\
\lambda=3 M_{4}^{2} H^{2}-6\left(\epsilon_{1} M_{5,2}^{3} \sqrt{H^{2}-H_{1}^{2}}+\epsilon_{2} M_{5,1}^{3} \sqrt{H^{2}-H_{2}^{2}}\right) \\
+4 M_{6}^{4} \frac{\cos \alpha}{\sin \alpha} .
\end{gathered}
$$

It is important to recognize that the induced gravity terms on the codimension-one branes provide contributions that are similar to the ones that appear in the DGP brane worlds in five dimensions. At the intersection, we find that localized gravity terms on the codimension-one branes induce contributions proportional to $M_{5, i}$ which are again similar to the ones in the DGP models in five dimensions. Note that, by allowing the different 5D Newton constant on different codimension-one branes, the parts of the Friedmann equation proportional to $M_{5, i}$ reproduce the asymmetric 5D model with induced gravity considered in Ref. [32]. In addition, we find a term inherited from the sixdimensional bulk that vanishes in the limit in which the branes form a right angle [26].

The angle between the branes can be determined by (3). In terms of the $H$ 's and the $\alpha$ it becomes

$$
\begin{aligned}
\Lambda_{B}= & -\frac{10}{\sin ^{2} \alpha}\left[H^{2}\left(1+\cos ^{2} \alpha\right)-H_{1}^{2}-H_{2}^{2}\right. \\
& \left.-2 \epsilon_{1} \epsilon_{2} \cos \alpha \sqrt{H^{2}-H_{1}^{2}} \sqrt{H^{2}-H_{2}^{2}}\right],
\end{aligned}
$$

that can be easily solved in terms of the angles:

\footnotetext{
${ }^{6}$ Notice that the six-dimensional terms proportional to $M_{6}$ have different coefficients with respect to the results in [26]. This discrepancy is due to a different prescription for manipulating distributional functions during the calculations. It does not qualitatively affect the physical consequences of the results.
}

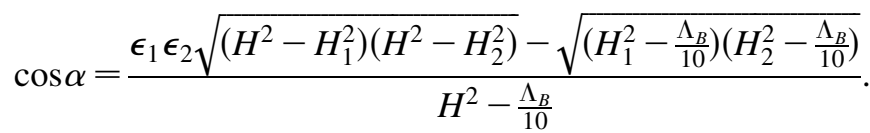

\section{SELF-ACCELERATING CONFIGURATIONS}

Potentially interesting configurations in our system are self-accelerating solutions. It is well known that induced gravity terms may provide accelerating cosmological solutions in which acceleration is induced by gravity itself and is independent of the presence of matter or cosmological constant in the system. This very important observation $[2,3]$ has received much attention, since it can provide a model for dark energy without cosmological constant. However, it has also been realized that this scenario has a serious drawback. The most interesting branch of solutions in the standard DGP brane world (the one that contains the self-accelerating configuration) is plagued by ghosts [5]. It would be interesting to study how generic this conclusion is in higher codimensional models. In order to investigate this issue, we start from seeking new self-accelerating solutions in higher codimensions.

\section{A. New self-accelerating solutions in 6D spacetime}

We consider now self-accelerating solutions for our system. Let us then choose $\Lambda_{1}=\Lambda_{2}=\lambda=0$, while the induced gravity terms (parametrized by $M_{4}$ and $M_{5, i}$ ) do not vanish. For simplicity we assume $M_{5,1}=M_{5,2}$. It is then possible to solve our system of equations (26)-(29). Taking the difference between (26) and (27), one obtains

$$
\begin{aligned}
&\left(\epsilon_{1} \sqrt{H^{2}-H_{1}^{2}}-\epsilon_{2} \sqrt{H^{2}-H_{2}^{2}}\right)\left[\frac{1+\cos \alpha}{\sin \alpha}\right. \\
&\left.-\frac{6 M_{5}^{3}}{8 M_{6}^{4}}\left(\epsilon_{1} \sqrt{H^{2}-H_{1}^{2}}+\epsilon_{2} \sqrt{H^{2}-H_{2}^{2}}\right)\right]=0,
\end{aligned}
$$

so that we have two possible types of solutions that we now describe.

(i) Symmetric solution: $H_{1}=H_{2}$ and $\epsilon_{1}=\epsilon_{2}$.-The flat-bulk condition (29) yields

$$
H_{1}^{2}=\frac{H^{2}}{2}(1-\cos \alpha) \text {. }
$$

From the codimension-one equation (26), one thus gets either the trivial solution $H=0$, or

$$
H=\frac{8 \epsilon_{1}}{3 \sin \alpha} \frac{M_{6}^{4}}{M_{5}^{3}} \sqrt{\frac{1+\cos \alpha}{2}}
$$

which, along with (32), can be plugged into the intersection equation (28) to give

$$
4+3 \cos \alpha=\frac{8 M_{6}^{4} M_{4}^{2}(1+\cos \alpha)}{3 M_{5}^{3} \sin \alpha},
$$


that sets the value for the angle between the codimension-one branes. Notice that this last relation forces the angle $\alpha$ to be positive. Then, in order to have a positive $H$, for Eq. (33) we must choose a positive $\epsilon$.

(ii) Asymmetric solution: $H_{1} \neq H_{2}$. - Defining

$$
\mathcal{D}=-\frac{6 M_{5}^{3}}{8 M_{6}^{4} \sin \alpha}\left(\epsilon_{1} \sqrt{H^{2}-H_{1}^{2}}+\epsilon_{2} \sqrt{H^{2}-H_{2}^{2}}\right),
$$

we get

$$
\mathcal{D}=\frac{1}{\cos \alpha-1},
$$

from (31). Then the sum of (26)-(28) gives

$$
\begin{gathered}
\sqrt{H_{1}^{2}+H_{2}^{2}}=\frac{4 M_{6}^{4}}{3 M_{5}^{3}}, \\
H^{2}=\frac{4 M_{6}^{4}}{3 M_{4}^{2}} \frac{2+\cos \alpha}{\sin \alpha},
\end{gathered}
$$

respectively. Finally from the condition (29) we get the relation

$$
\sin \alpha(2+\cos \alpha)=\frac{4 M_{6}^{4} M_{4}^{2}}{3 M_{5}^{6}} .
$$

From this condition, we learn that $\alpha$ is positive and then, from the previous relations, that at least one of the $\epsilon$ 's must be positive as well.

\section{B. Scales of gravity}

A simple manipulation of the previous formulas shows that, in both cases, the resulting Hubble parameter is of the order

$$
H \simeq \frac{M_{5}^{3}}{M_{4}^{2}},
$$

that is, the same result as in the five-dimensional DGP models. In order to explain the late time acceleration $H \sim$ $10^{-42} \mathrm{GeV}$, we should require $M_{5}=10^{-2} \mathrm{GeV}$ with $M_{4}=10^{18} \mathrm{GeV}$. For $\alpha \sim \pi / 2$, the six-dimensional Newton constant is roughly given by

$$
M_{6}^{4} \simeq \frac{M_{5}^{6}}{M_{4}^{2}} \simeq M_{4}^{2} H^{2} .
$$

Thus, $M_{6}$ must be $M_{6}=10^{-3} \mathrm{eV}$ to explain the late time acceleration. With these parameters we find that the crossover scales are given by

$$
r_{c, 5} \sim r_{c, 6} \sim H^{-1}, \quad r_{c, 5}=\frac{M_{4}^{2}}{M_{5}^{3}}, \quad r_{c, 6}=\frac{M_{4}}{M_{6}^{2}} .
$$

We should note that these scales are exactly the seesaw scale of [33]. For large distance, $r>r_{c, 5}, r_{c, 6}$, gravity is five-dimensional or six-dimensional. The laws of fourdimensional gravity are valid all the way down to the distances of the order $M_{6}^{-1}$. Below this length scale, the effective theory of gravity breaks down. As a result, there is a lower bound on the scale $M_{6}$ which comes from accelerators, astroparticle, and cosmological data, that is $M_{6}>$ $10^{-3} \mathrm{eV}$ [34]. This is exactly the scale we need to explain the present-day acceleration of the Universe. Note that, for a small angle $\alpha \ll 1, M_{6}$ is given by $M_{6}^{4}=\alpha M_{5}^{6} / M_{4}^{2}$. Hence, the constraint becomes difficult to satisfy.

However, one realizes that, in order to have $H$ positive, we must choose at least one of the $\epsilon_{i}$ with the positive sign. Then, we are in the same class of self-accelerating solutions of [2], that are notoriously plagued by ghosts. It is then likely that our codimension-one branes contain ghost excitations. There is a suggestion that, if there is no matter on the self-accelerating universe, it is possible to quantize the theory without having the ghost instability. In our model, we do not need matter on codimension-one brane. It would be interesting to study at what level the ghost couples with matter on the brane at the intersection.

\section{More self-accelerating solutions in higher codimensions}

In this section we show that the methods previously described can be easily generalized to a system of $N$ codimension-one branes that intersect in a $(4+N)$ dimensional spacetime, along the lines of [35]. Their common intersection - a four-dimensional, codimension- $N$ brane-should correspond to the spacetime we observe. We consider an Einstein-Hilbert action in the bulk, with the addition of a bulk Lagrangian $L$, and we allow for induced gravity terms on all the branes, and at the intersections.

The action for the system is then the following:

$$
\begin{aligned}
S= & \int_{\text {bulk }} d^{4+N} x \sqrt{-g}\left(\frac{M_{4+N}^{2+N}}{2} R+L\right) \\
& +\sum_{i=1}^{N} \int_{\Sigma_{i}} d^{3+N} x \sqrt{-g_{(i)}}\left(\frac{M_{3+N}^{1+N}}{2} R_{(i)}+L_{(i)}\right) \\
& +\sum_{i \neq j} \int_{\Sigma_{i} \cap \Sigma_{j}} d^{2+N} x \sqrt{-g_{(i j)}}\left(\frac{M_{2+N}^{N}}{2} R_{(i j)}+L_{(i j)}\right) \\
& +\cdots+\int_{\cap} d^{4} x \sqrt{-g_{\cap}}\left(\frac{M_{4}^{2}}{2} R_{\cap}+L_{\cap}\right),
\end{aligned}
$$

where $\cap \equiv \bigcap_{i} \Sigma_{i}$ denotes the three-brane at the intersection between all codimension-one branes. Here we concentrate on the case where the Lagrangians are simply tensions and the codimension-one branes intersect to form right angles. We also describe an empty "self-accelerated" scenario where all the branes undergo a de Sitter phase with no cosmological constant nor tensions in the system. The bulk 
geometry is again a maximally symmetric one: $d s^{2}=$ $A^{2}\left(t, z_{1}, \ldots, z_{N}\right) \eta_{M N} d x^{M} d x^{N} \quad$ with $\quad A\left(t, z_{1}, \ldots, z_{N}\right)=$ $1 / H t+\sum_{i=1}^{N} k_{i}\left|z_{i}\right|$ and each static codimension-one brane $\Sigma_{i}$ is defined by the subspace $\left\{z_{i}=0\right\}$. The bulk Einstein equation gives the relation

$$
H^{2}-\sum_{i=1}^{N} k_{i}^{2}=\frac{2}{(2+N)(3+N)} \Lambda,
$$

whereas the equation of motion for the codimension-one branes $\Sigma_{i}$ can be computed by means of the Israel junction condition. Assuming $Z_{2}$ symmetry across the branes

$$
\text { (i) } K_{n}^{m}=-\left.\frac{1}{2 M_{4+N}^{2+N}}\left({ }^{(i)} T^{m}{ }_{n}-\frac{1}{2+N} \delta_{n}^{m(i)} T\right)\right|_{\text {smooth }},
$$

where ${ }^{(i)} T^{m}{ }_{n}$ includes a regular "matter" part $-\Lambda_{(i)} \delta_{n}^{m}$ and a part from the smooth part of the induced EH term, namely $-M_{3+N}^{1+N(i)} G_{n}^{m}=M_{3+N}^{1+N} \frac{(1+N)(2+N)}{2}\left(H^{2}-\sum_{i^{\prime}} k_{i^{\prime}}^{2}\right) \delta_{n}^{m}$. Hence,

$$
\begin{aligned}
\left.{ }^{(i)} T^{m}{ }_{n}\right|_{\text {smooth }}= & \left(-\Lambda_{(i)}+M_{3+N}^{1+N} \frac{(1+N)(2+N)}{2}\right. \\
& \left.\times\left(H^{2}-\sum_{i^{\prime}} k_{i^{\prime}}^{2}\right)\right) \delta_{n}^{m} .
\end{aligned}
$$

Taking the normal vector to the brane to be pointing into the bulk $n_{M}^{+}=A \delta_{M}^{z_{i}}$, we get ${ }^{(i)} K^{m}{ }_{n}=g^{m r} \nabla_{r} n_{n}=$ $\left.\delta_{n}^{m} A^{-2} \partial_{i} A\right|_{\Sigma_{i}}=-\delta_{n}^{m} k_{i}$. Then Eq. (45) gives

$$
\begin{aligned}
\Lambda_{i}= & M_{3+N}^{1+N} \frac{(1+N)(2+N)}{2}\left(H^{2}-\sum_{\ell \neq i} k_{\ell}^{2}\right) \\
& +2(2+N) M_{4+N}^{2+N} k_{i},
\end{aligned}
$$

which in the special case $N=2$ simply reduces to (26) for a right angle between the branes. The process can be iterated. In fact each brane $\Sigma_{i}$ is intersected by other $N-$ 1 branes. From the point of view of an observer sitting on $\Sigma_{i}$, each of these intersections separate the brane itself in two half parts whose boundaries are given by that intersection. Consider for definiteness the intersection $\Sigma_{i} \cap \Sigma_{j}$ : we will then have a contribution to the singular part of the Einstein tensors associated to $R_{(i)}$ localized at $z_{j}=0$ and a contribution to the singular part of the Einstein tensors associated to $R_{(j)}$ localized at $z_{i}=0$. Such contributions can be evaluated by computing the extrinsic curvatures associated to the intersection $\Sigma_{i} \cap \Sigma_{j}$ as measured from the $\Sigma_{i}$ and $\Sigma_{j}$ point of view. Namely,

$$
{ }^{(i j)} K_{n}^{m}+{ }^{(j i)} K_{n}^{m}=-\delta_{n}^{m}\left(k_{i}+k_{j}\right),
$$

and (47) thus generalizes as

$$
\begin{aligned}
\Lambda_{(i j)}= & M_{2+N}^{N} \frac{N(1+N)}{2}\left(H^{2}-\sum_{l \neq i, j} k_{l}^{2}\right) \\
& +2(1+N) M_{3+N}^{1+N}\left(k_{i}+k_{j}\right),
\end{aligned}
$$

and so on. In particular, for the 3-brane intersection of all the codimension-one branes, we have $N$ contributions from the induced $\mathrm{EH}$ terms localized on the $N-1$-codimension $N$ intersections. Hence,

$$
\Lambda_{\cap}=3 M_{4}^{2} H^{2}+6 M_{5}^{3} \sum_{i=1}^{N} k_{i} .
$$

It is thus clear from the previous setup that the presence of induced EH terms on the branes allows for localized matter on the branes themselves, at least in the form of tension.

We then concentrate on a special case of the previous results that gives rise to a de Sitter solution in the absence of any form of matter in the system, namely, a selfaccelerated solution. We set $k_{i}=-H_{i}$ : the bulk equation of motion simply requires $H^{2}=\sum_{i} H_{i}^{2}$, whereas from the junctions conditions (47) one obtains

$$
M_{3+N}^{1+N} \frac{(1+N)}{4}\left(H^{2}-\sum_{\ell \neq i} H_{\ell}^{2}\right)=M_{4+N}^{2+N} H_{i},
$$

that, using the bulk equation of motion, reduces to

$$
H_{i}=\frac{M_{3+N}^{1+N}}{M_{4+N}^{2+N}} \frac{1+N}{4} H_{i}^{2}
$$

which fixes

$$
K \equiv H_{i}=\frac{4}{1+N} \frac{M_{4+N}^{2+N}}{M_{3+N}^{1+N}}, \quad \forall i .
$$

The process can again be iterated all the way down to codimension- $N$. At the last level, we have $N$ contributions to the extrinsic curvature on the 3-brane, from $N$ possible intersections of $N-1$ codimension-one branes. Using (50) and (53) and the bulk equation of motion, one obtains the 4D Hubble scale and a relation between all the masses in the system:

$$
\begin{gathered}
H=\sqrt{N} K=\sqrt{N} 2 \frac{M_{5}^{3}}{M_{4}^{2}}, \\
2 \frac{M_{5}^{3}}{M_{4}^{2}}=\frac{4}{3} \frac{M_{6}^{4}}{M_{5}^{3}}=\frac{M_{7}^{5}}{M_{6}^{4}}=\cdots=\frac{4}{1+N} \frac{M_{4+N}^{2+N}}{M_{3+N}^{1+N}} .
\end{gathered}
$$

Let us also stress that all the branes (codimension-one and intersections) undergo a de Sitter phase. As to the codimension-one branes $\Sigma_{i}$, the rate is simply $H_{i}=K$, and similarly for $\Sigma_{i} \cap \Sigma_{j}$ one has $H_{i j}=\sqrt{H_{i}^{2}+H_{j}^{2}}=$ $\sqrt{2} K$ : for a generic codimension- $n$ intersection, one obtains $H_{(n)}=\sqrt{n} K$. 
Note that if we require the de Sitter rate $H$ to be equal to today's Hubble rate $H_{0} \sim 10^{-42} \mathrm{GeV}$, from (54) and (55), and using $M_{4} \sim 10^{18} \mathrm{GeV}$, we get the following values for the mass scales involved in the system:

$$
\begin{gathered}
M_{5} \sim 10^{-2} \mathrm{GeV}, \quad M_{6} \sim M_{5}\left(\frac{M_{5}}{M_{4}}\right)^{1 / 2} \sim 10^{-12} \mathrm{GeV}, \\
M_{7} \sim M_{6}\left(\frac{M_{6}}{M_{5}}\right)^{3 / 5} \sim 10^{-18} \mathrm{GeV} \ldots,
\end{gathered}
$$

Hence the higher is the codimension, the lower the fundamental scale must be. This requirement contradicts the constraints on the fundamental scale $M_{4+N}<10^{-3} \mathrm{eV}$, and it is impossible to realize the self-accelerated scenarios in higher codimensional spacetime with $N>2$.

\section{SELF-TUNING CONFIGURATIONS}

The system of equations (26)-(29) relates the bulk cosmological constant and brane tensions with the brane Hubble parameters and the angle between the branes. Since we have induced gravity terms on the branes, it is possible that they are enough to ensure that four-dimensional gravity is obtained in the relevant regimes at the intersection, with no need to compactify the extra dimensions. Then, the angle between the branes is a free parameter; it is natural to ask whether the self-tuning solutions exist where, by changing the tension at the intersection $\lambda$, only the angle $\alpha$ between the branes changes and all the other quantities are fixed.

The following is an example of such solutions:

$$
\begin{gathered}
\Lambda_{1}=6 M_{5,1}^{3} H_{1}^{2}, \\
\Lambda_{2}=6 M_{5,2}^{3} H_{2}^{2}, \\
H^{2}=H_{1}^{2}=H_{2}^{2}=\frac{\Lambda_{B}}{10}, \\
\cos ^{2} \alpha=\frac{\left(\lambda-3 M_{4}^{2} H^{2}\right)^{2}}{\left(\lambda-3 M_{4}^{2} H^{2}\right)^{2}+16 M_{6}^{8}} .
\end{gathered}
$$

The Hubble parameter at the intersection is independent of the tension $\lambda$. The tension only controls the angle between the branes. Then, if we localize standard model physics at the intersection, any contribution to the four-dimensional cosmological constant, that is, the brane tension $\lambda$, does not curve the four-dimensional spacetime. Instead, it only changes a parameter in the higher dimensional spacetime that is the angle between the branes, realizing the desired self-tuning mechanism.

It is important to point out the similarity and difference between this idea and the self-tuning scenarios in six dimensions using brane worlds on conical singularities [7,9] where two extra dimensions are compactified by a magnetic monopole. In these cases, the self-tuning mechanism suffers from a quantization condition for the magnetic monopole responsible for compactifying the extra space and we need a fine-tuning between the cosmological constant and other parameters of the models. Our case is different because in principle we do not need to compactify the extra dimensions. In our model, the induced gravity is supposed to recover 4D behaviors of gravity on scales smaller than the crossover scales $r_{c, 5}$ or $r_{c, 6}$. Furthermore, note that in [7,9] and in their infinite-volume counterparts [11,15] (which are more like the present configuration), the vacuum energy density has a finite critical value that corresponds to the maximum of the deficit angle of the cone that characterizes the 6D spacetime near the location of the brane. Our solution is topologically different from a vortex and there is no critical value for the brane tension. This would have an important implication for the existence of the ghost. It has been shown that there appears a ghost if we consider a 4D vortex with induced gravity in a 6D spacetime depending on the regularizations [20]. In our model, we should carefully reexamine this issue as the intersection behaves differently from the vortex. It would be interesting to study this topic in more detail.

\section{CONCLUSIONS AND OPEN ISSUES}

In this paper, we studied maximally symmetric solutions for intersecting brane worlds with induced gravity terms. We found new classes of the self-accelerating solutions that realize de Sitter solution without any cosmological constant in the system. In a $6 \mathrm{D}$ spacetime, this realizes the seesaw modification of the gravity. The $6 \mathrm{D}$ Planck scale must be $M_{6}=10^{-3} \mathrm{eV}$ to explain the late time acceleration $H=H_{0}=10^{-42} \mathrm{GeV}$. This scale determines the IR scale where 4D gravity is modified $M_{6}^{2} / M_{4} \sim H_{0}$. On the other hand, $M_{6}$ defines the UV scale where $4 \mathrm{D}$ gravity is modified. It was shown that the lower bound for the fundamental Planck scale is roughly $10^{-3} \mathrm{eV}$ from various constraints which is consistent in the 6D case. The small angle between the two branes makes it difficult to satisfy this constraint. We have shown that the construction of the self-accelerating universe with right angles between the branes can be naturally extended to $N$-dimensional spacetime. However, it was shown that, in order to realize the observed late time acceleration, the higher-dimensional Planck scale is increasingly lower and violates the lower bound for the fundamental scale. We should mention that the problem of the ghost instability would exist even in these new solutions. It would be important to study at what level the ghost couples with matter on the brane at the intersection.

It is also possible to find the self-tuning solution where the cosmological constant on the intersection only depends on the angle between the branes. Unlike other examples, in principle we do not need to compactify the extra dimensions, since the induced gravity terms may be sufficient to provide four-dimensional gravity in the relevant regimes. 
Then, the angle between the branes is a free parameter that does not have to satisfy any constraint. We also showed that a brane world at the intersection behaves differently from the brane world on a vortex. In the vortex case, it is shown that a regularization is needed to remove the divergence of 6D propagator and a ghost can appear depending on the regularization. It is vital to reexamine these issues for our intersecting branes.

Finally, let us briefly discuss the problem of obtaining consistent four-dimensional gravity at the intersection in this setup. The first simple step toward the understanding of this issue is to generalize the maximally symmetric solutions to the case of FRW cosmological configurations on the branes. Then, by studying Israel junction conditions, one can derive the effective cosmological equations on the codimension-one and codimension-two brane worlds. By obtaining the Hubble equation on the codimension-two brane world at the intersection, it is possible to determine on which scales standard four-dimensional cosmological expansion can be obtained at the intersection. The resulting cosmological system presents many interesting features. It is possible to show that the preferred energy-momentum tensor can be localized at the intersection, although in the case in which it is different from pure tension, there is normally an exchange of energy density between the codimension-one branes and the intersection. The details of the induced cosmology, as well as their consequences, are going to be analyzed in a companion paper [27].

\section{ACKNOWLEDGMENTS}

We thank Hyun Min Lee and Nemanja Kaloper for useful discussions. K. K. is supported by STFC. G. T. is supported by MEC and FEDER under Grant No. FPA200605485, by CAM under Grant No. HEPHACOS P-ESP00346, and by the "UniverseNet" network (MRTN-CT2006-035863). O. C. and G. T. are grateful to the Institute of Cosmology and Gravitation of the University of Portsmouth for support and warm hospitality while this work was initiated.
[1] G. R. Dvali, G. Gabadadze, and M. Porrati, Phys. Lett. B 485, 208 (2000).

[2] C. Deffayet, Phys. Lett. B 502, 199 (2001).

[3] C. Deffayet, G. R. Dvali, and G. Gabadadze, Phys. Rev. D 65, 044023 (2002).

[4] K. Koyama, Gen. Relativ. Gravit. 40, 421 (2008).

[5] M. A. Luty, M. Porrati, and R. Rattazzi, J. High Energy Phys. 09 (2003) 029; A. Nicolis and R. Rattazzi, J. High Energy Phys. 06 (2004) 059; K. Koyama, Phys. Rev. D 72, 123511 (2005); D. Gorbunov, K. Koyama, and S. Sibiryakov, Phys. Rev. D 73, 044016 (2006); C. Charmousis, R. Gregory, N. Kaloper, and A. Padilla, J. High Energy Phys. 10 (2006) 066; C. Deffayet, G. Gabadadze, and A. Iglesias, J. Cosmol. Astropart. Phys. 08 (2006) 012; K. Izumi, K. Koyama, and T. Tanaka, J. High Energy Phys. 04 (2007) 053; K. Izumi, K. Koyama, O. Pujolas, and T. Tanaka, Phys. Rev. D 76, 104041 (2007); K. Koyama and F. P. Silva, Phys. Rev. D 75, 084040 (2007); K. Koyama, Classical Quantum Gravity 24, R231 (2007); R. Gregory, N. Kaloper, R. C. Myers, and A. Padilla, J. High Energy Phys. 10 (2007) 069.

[6] J. W. Chen, M. A. Luty, and E. Ponton, J. High Energy Phys. 09 (2000) 012.

[7] S. M. Carroll and M. M. Guica, arXiv:hep-th/0302067; I. Navarro, J. Cosmol. Astropart. Phys. 09 (2003) 004; Y. Aghababaie, C. P. Burgess, S. L. Parameswaran, and F. Quevedo, Nucl. Phys. B680, 389 (2004).

[8] Y. Aghababaie et al., J. High Energy Phys. 09 (2003) 037; H. M. Lee and A. Papazoglou, Nucl. Phys. B747, 294 (2006); B765, 200(E) (2007); C.P. Burgess, C. de Rham, D. Hoover, D. Mason, and A. J. Tolley, J. Cosmol. Astropart. Phys. 02 (2007) 009; A. J. Tolley, C. P. Burgess, C. de Rham, and D. Hoover, New J. Phys.
8, 324 (2006); A. J. Tolley, C. P. Burgess, D. Hoover, and Y. Aghababaie, J. High Energy Phys. 03 (2006) 091; C. P. Burgess, arXiv:hep-th/0510123; S. L. Parameswaran, G. Tasinato, and I. Zavala, Nucl. Phys. B737, 49 (2006).

[9] Y. Aghababaie, C. P. Burgess, S. L. Parameswaran, and F. Quevedo, Nucl. Phys. B680, 389 (2004).

[10] R. Gregory, V. A. Rubakov, and S. M. Sibiryakov, Phys. Rev. Lett. 84, 5928 (2000).

[11] G. R. Dvali and G. Gabadadze, Phys. Rev. D 63, 065007 (2001); O. Corradini, A. Iglesias, Z. Kakushadze, and P. Langfelder, Phys. Lett. B 521, 96 (2001); G. Dvali, G. Gabadadze, and M. Shifman, Phys. Rev. D 67, 044020 (2003); arXiv:hep-th/0208096.

[12] S. Kachru, M. B. Schulz, and E. Silverstein, Phys. Rev. D 62, 045021 (2000); N. Arkani-Hamed, S. Dimopoulos, N. Kaloper, and R. Sundrum, Phys. Lett. B 480, 193 (2000); S. Forste, Z. Lalak, S. Lavignac, and H. P. Nilles, Phys. Lett. B 481, 360 (2000); J. High Energy Phys. 09 (2000) 034.

[13] I. Navarro, Classical Quantum Gravity 20, 3603 (2003); H. P. Nilles, A. Papazoglou, and G. Tasinato, Nucl. Phys. B677, 405 (2004); J. Garriga and M. Porrati, J. High Energy Phys. 08 (2004) 028; H. M. Lee, Phys. Lett. B 587, 117 (2004).

[14] J. M. Cline, J. Descheneau, M. Giovannini, and J. Vinet, J. High Energy Phys. 06 (2003) 048; J. Vinet and J. M. Cline, Phys. Rev. D 70, 083514 (2004); 71, 064011 (2005).

[15] P. Bostock, R. Gregory, I. Navarro, and J. Santiago, Phys. Rev. Lett. 92, 221601 (2004); S. Kanno and J. Soda, J. Cosmol. Astropart. Phys. 07 (2004) 002.

[16] G. Kofinas, Phys. Lett. B 633, 141 (2006); E. Papantonopoulos and A. Papazoglou, J. High Energy Phys. 09 (2005) 012; J. Cosmol. Astropart. Phys. 07 
(2005) 004.

[17] M. Peloso, L. Sorbo, and G. Tasinato, Phys. Rev. D 73, 104025 (2006).

[18] N. Kaloper, Phys. Lett. B 652, 92 (2007); C. P. Burgess, D. Hoover, and G. Tasinato, J. High Energy Phys. 09 (2007) 124; C. P. Burgess, F. Quevedo, G. Tasinato, and I. Zavala, J. High Energy Phys. 11 (2004) 069; F. Arroja, T. Kobayashi, K. Koyama, and T. Shiromizu, J. Cosmol. Astropart. Phys. 12 (2007) 006; E. Papantonopoulos, A. Papazoglou, and V. Zamarias, arXiv:0707.1396; M. Minamitsuji and D. Langlois, Phys. Rev. D 76, 084031 (2007); A. J. Tolley, C. P. Burgess, C. de Rham, and D. Hoover, New J. Phys. 8, 324 (2006); B. Himmetoglu and M. Peloso, Nucl. Phys. B773, 84 (2007); T. Kobayashi and M. Minamitsuji, J. Cosmol. Astropart. Phys. 07 (2007) 016; E. J. Copeland and O. Seto, J. High Energy Phys. 08 (2007) 001; T. Kobayashi and Y.i. Takamizu, arXiv:0707.0894.

[19] E. Kiritsis, N. Tetradis, and T. N. Tomaras, J. High Energy Phys. 08 (2001) 012; C. Charmousis and R. Zegers, J. High Energy Phys. 08 (2005) 075; Phys. Rev. D 72, 064005 (2005); I. Navarro and J. Santiago, J. High Energy Phys. 02 (2005) 007; C. de Rham, J. High Energy Phys. 01 (2008) 060.

[20] S. L. Dubovsky and V.A. Rubakov, Phys. Rev. D 67, 104014 (2003); M. Kolanovic, M. Porrati, and J. W. Rombouts, Phys. Rev. D 68, 064018 (2003); G. Gabadadze and M. Shifman, Phys. Rev. D 69, 124032 (2004).

[21] N. Kaloper and D. Kiley, J. High Energy Phys. 05 (2007) 045; N. Kaloper, arXiv:0711.3210.

[22] N. Arkani-Hamed, S. Dimopoulos, G. R. Dvali, and N. Kaloper, Phys. Rev. Lett. 84, 586 (2000); C. Csaki and Y. Shirman, Phys. Rev. D 61, 024008 (1999); A. E. Nelson, Phys. Rev. D 63, 087503 (2001); J. Cline, C. Grojean, and
G. Servant, Phys. Lett. B 472, 302 (2000).

[23] H. M. Lee and G. Tasinato, J. Cosmol. Astropart. Phys. 04 (2004) 009.

[24] J.E. Kim, B. Kyae, and H. M. Lee, Phys. Rev. D 64, 065011 (2001); J.E. Kim and H. M. Lee, Phys. Rev. D 65, 026008 (2001).

[25] R. Blumenhagen, L. Goerlich, B. Kors, and D. Lust, J. High Energy Phys. 10 (2000) 006; G. Aldazabal, S. Franco, L. E. Ibanez, R. Rabadan, and A. M. Uranga, J. Math. Phys. (N.Y.) 42, 3103 (2001); L. E. Ibanez, F. Marchesano, and R. Rabadan, J. High Energy Phys. 11 (2001) 002; G. Aldazabal, S. Franco, L. E. Ibanez, R. Rabadan, and A. M. Uranga, J. High Energy Phys. 02 (2001) 047; C. Kokorelis, J. High Energy Phys. 08 (2002) 018.

[26] N. Kaloper, J. High Energy Phys. 05 (2004) 061.

[27] O. Corradini, K. Koyama, and G. Tasinato (unpublished).

[28] C. de Rham, G. Dvali, S. Hofmann, J. Khoury, O. Pujolas, M. Redi, and A. J. Tolley, arXiv:0711.2072.

[29] R. Gregory and A. Padilla, Phys. Rev. D 65, 084013 (2002); G. Dvali, G. Gabadadze, O. Pujolas, and R. Rahman, Phys. Rev. D 75, 124013 (2007).

[30] I. Antoniadis, Lect. Notes Phys. 720, 293 (2007); I. Antoniadis, R. Minasian, and P. Vanhove, Nucl. Phys. B648, 69 (2003).

[31] E. Kohlprath, Nucl. Phys. B697, 243 (2004); F. T. J. Epple, J. High Energy Phys. 09 (2004) 021.

[32] C. Charmousis, R. Gregory, and A. Padilla, J. Cosmol. Astropart. Phys. 10 (2007) 006.

[33] G. Dvali, G. Gabadadze, X.r. Hou, and E. Sefusatti, Phys. Rev. D 67, 044019 (2003).

[34] G. R. Dvali, G. Gabadadze, M. Kolanovic, and F. Nitti, Phys. Rev. D 65, 024031 (2001).

[35] I. Navarro and J. Santiago, J. High Energy Phys. 04 (2004) 062. 